SUBJECT AREAS:

CELL BIOLOGY

STEM CELLS

Received

20 September 2013

Accepted

27 December 2013

Published

21 January 2014

Correspondence and requests for materials should be addressed to

T.-S.L. (litaoshe@ nagasaki-u.ac.jp)

\section{Effects of antioxidants on the quality and genomic stability of induced pluripotent stem cells}

Lan Luo', ', Miho Kawakatsu', Chao-Wan Guo', Yoshishige Urata', Wen-Jing Huang' ', Haytham Ali', Hanako Doi' ', Yuriko Kitajima', Takayuki Tanaka', Shinji Goto', Yusuke Ono' ', Hong-Bo Xin ${ }^{3}$, Kimikazu Hamano² \& Tao-Sheng Li'

'Department of Stem Cell Biology, Atomic Bomb Disease Institute, Nagasaki University, 1-12-4 Sakamoto, Nagasaki 852-8523, Japan, ${ }^{2}$ Department of Surgery and Clinical Sciences, Yamaguchi University Graduate School of Medicine, 1-1-1 Minami-Kogushi, Ube, Yamaguchi 755-8505, Japan, ${ }^{3}$ Institute of Translational Medicine, Nanchang University, Nanchang, Jiangxi 330031, PR China.

Effects of antioxidants on the quality and genomic stability of induced pluripotent stem (iPS) cells were investigated with two human iPS cell lines (201B7 and 253G1). Cells used in this study were expanded from a single colony of each cell line with the addition of proprietary antioxidant supplement or homemade antioxidant cocktail in medium, and maintained in parallel for 2 months. The cells grew well in all culture conditions and kept "stemness". Although antioxidants modestly decreased the levels of intracellular reactive oxygen species, there were no differences in the expression of 53BP1 and pATM, two critical molecules related with DNA damage and repair, under various culture conditions. CGH analysis showed that the events of genetic aberrations were decreased only in the 253G1 iPS cells with the addition of homemade antioxidant cocktail. Long-term culture will be necessary to confirm whether low dose antioxidants improve the quality and genomic stability of iPS cells.

\footnotetext{
hromosomal aberrations are commonly found in stem cells after long-term cultivation in a general $\mathrm{CO}_{2}$ incubator where the media was equilibrated with $95 \%$ air and $5 \% \mathrm{CO}_{2}\left(\sim 20 \% \mathrm{O}_{2}\right)$, which is much higher than in the in vivo physiological microenvironment of the stem cell niche $\left(\sim 1-5 \% \mathrm{O}_{2}\right.$, depending on the tissue $)^{1-4}$. The exposure of stem cells to a non-physiological hyperoxic state may lead to oxidative stress and induce DNA damage ${ }^{5,6}$. A number of studies have recently tried to improve the genomic stability of stem cells by culturing stem cells under physiological lower oxygen ${ }^{7-10}$. However, these cells will be exposed to air during the experimental processes, such as the medium change and cell passaging, unless a special oxygen-controllable clean bench is available.

Alternatively, the addition of antioxidants in medium may effectively attenuate oxidative stress-induced genomic instability of stem cells during in vitro expansion. Although the basic culture medium is well-known to be consist of many amino acids and vitamins, and some supplements specially for stem cell culture are also contained antioxidants, it still keeps unclear whether the basal level of antioxidants in medium is enough or not. Interestingly, we have recently discovered a biphasic effect of antioxidants on genomic stability of stem cells ${ }^{9}$. We found that the supplement of low dosages of antioxidant cocktails likely contribute to the decrease DNA damage and the improvement of genomic stability of stem cells, conversely, high dosages of antioxidants increase the risk of chromosomal abnormalities of stem cells by interfering with the endogenous DNA repair pathways.

Herein, we examined whether the supplement of low dosages of antioxidants in culture medium could improve the quality and genomic stability of induced pluripotent stem (iPS) cells during long-term ex vivo expansion.
}

\section{Results}

Low dose antioxidants did not affect the growth and "stemness" of iPS cells. We successfully maintained the iPS cell lines for 2 months by regularly passage. The shape and growth of iPS cell colonies were not obviously changed by adding either proprietary antioxidant supplement from Sigma-Aldrich (AOS) or homemade antioxidant cocktail $(\mathrm{AOH})$ at relative low concentrations in culture medium for 2 months of follow-up. Immunostaining showed that all of these iPS cell colonies clearly expressed Oct3/4, Nanog, SSEA-4, and ALP 

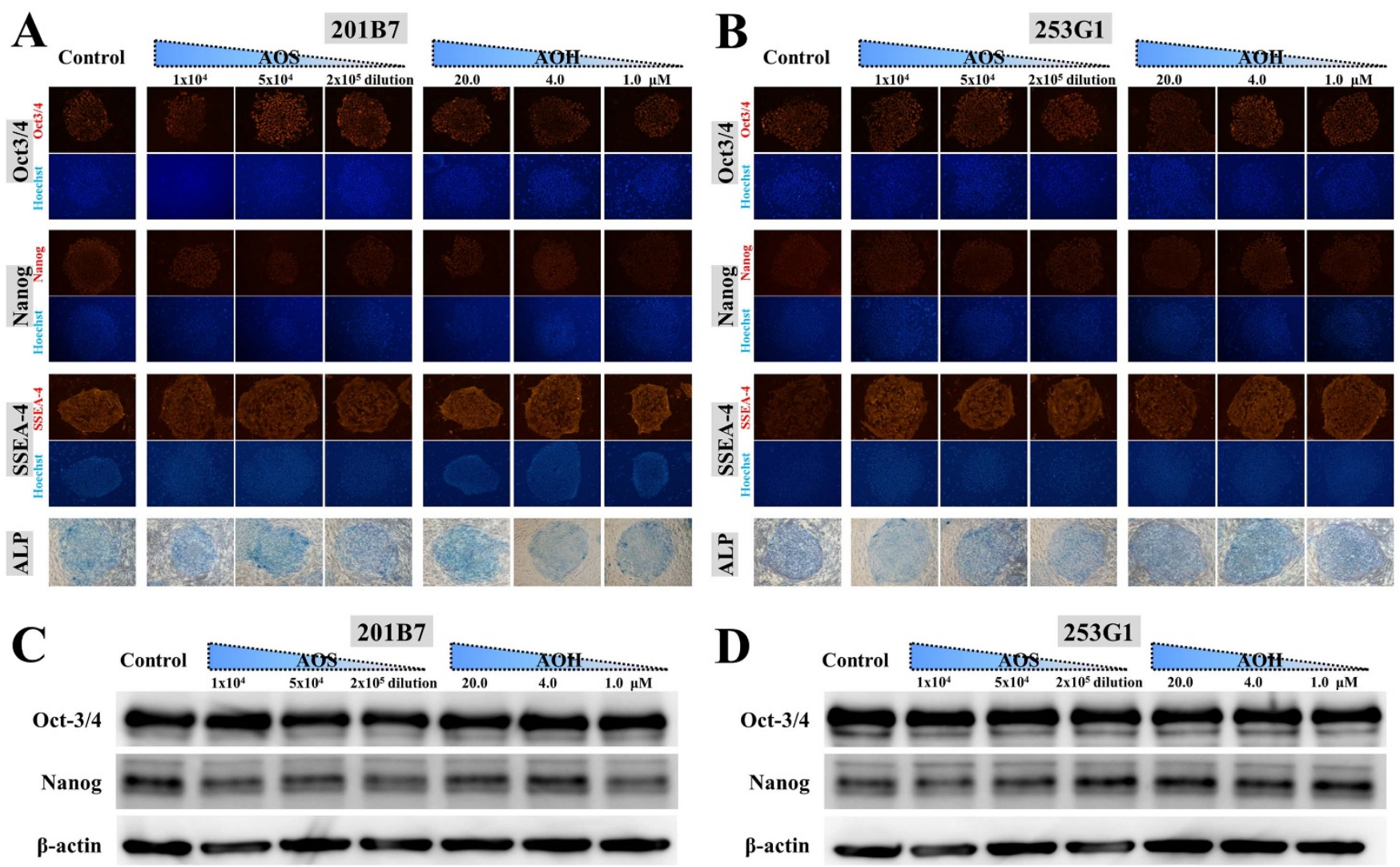

Figure $1 \mid$ Stemness of iPS cells after 2 months of culture. The expression of stem cell markers Oct3/4, Nanog, SSEA-4, and ALP were detected by staining, and representative images of expressions in 201B7 (A) and 253G1 (B) iPS cell lines were shown. Western blot analysis on the expressions of Nanog and Oct3/4 in 201B7 (C) and 253G1 (D) iPS cell lines was also done, and representative images that cropped from full-length blots (Supplementary Figure 1) was shown. Abbreviations: AOS, proprietary antioxidant supplement from Sigma-Aldrich; AOH, Homemade antioxidant cocktail.

after 2 months (Figure $1 \mathrm{~A}$ and $\mathrm{B}$ ), indicating that all culture conditions maintained "stemness" of iPS cells very well. Western blot analysis also showed that the expressions of Nanog and Oct3/ 4 at comparable high levels in all iPS cells under different culture conditions (Figure 1C and D), although the expressions were not carefully quantified.

Low dose antioxidants decreased the intracellular ROS levels in iPS cells. We first measured ROS level by detecting the fluorescence intensity under microscope (Figure 2A). When compared with the control, the addition of proprietary antioxidant supplement from Sigma-Aldrich or homemade antioxidant cocktail at relative low concentrations in culture medium obviously decreased the levels of intracellular ROS in the iPS cells (upper images in Figure 2A). Semiquantitative analysis showed that the relative fluorescence intensity of intracellular ROS were significantly lower in the iPS cells cultured with the addition of antioxidants in medium than that of the control (lower bar graphs in Figure 2A).

To further quantitative measure the ROS levels, we measured the fluorescence intensity in iPS cells by flow cytometry (Figure 2B). Again, the addition of antioxidants in medium showed to significantly decrease the ROS levels in the iPS cells, although the decrease of ROS by antioxidants was not clearly shown in a dose-dependent manner.

Low dose antioxidants did not promote DNA damage or inhibit DNA repair in iPS cells. We evaluated the DNA damage by counting the formation of 53BP1 foci in the nuclei of iPS cells after 2 months culture with the addition of antioxidants in medium or without. A quantitative analysis showed that the percentages of iPS cells with 53BP1 foci (Figure 3A,B) in the nuclei, and the expressions of phosphorylated ATM measured by Western blotting (Figure 3C,D) were not notably different among culture conditions.

Genomic aberrations in iPS cells after 2 months culture. To facilitate direct comparisons, the same iPS cells that had been expanded from a single colony were used to initiate cultures under different conditions in parallel. The data from the array CGH showed some amplifications (red dots) and a few of deletions (green dots), with log2 ratios over 0.75 (Figure $4 \mathrm{~A}$, Supplementary Table 1). Compared with the control group which was not added antioxidants in medium, the events of genomic aberrations in the 201B7 cell line were unexpectedly observed when the addition of 10,000- and 200,000-fold diluted proprietary antioxidant supplement and $1 \mu \mathrm{M}$ homemade antioxidant cocktail (Figure 4B). Interestingly, the events of genomic aberrations in the $253 \mathrm{G} 1$ cell line were much lower with the addition of homemade antioxidant cocktail, but no obvious change by the addition of the proprietary antioxidant supplement (Figure 4B).

The PANTHER classification system revealed that the aberrant gene/proteins could be classified into twenty-five groups based on their molecular function (Figure 5). According to the data, the decreased chromosomal aberrations in the 253G1 cell line by the addition of homemade antioxidant cocktail were most likely classified as enzyme modulator, hydrolase, nucleic acid binding, receptor, and transcription factor (Figure 5). According to the biological process, we noted that these chromosomal aberrations were likely associated with cell communication, cellular process, and metabolic processes in both cell lines (Figure 6, Supplementary Table 2).

\section{Discussion}

In this study, we examined whether the addition of low dose antioxidants in culture medium affects the growth, quality, and genomic 

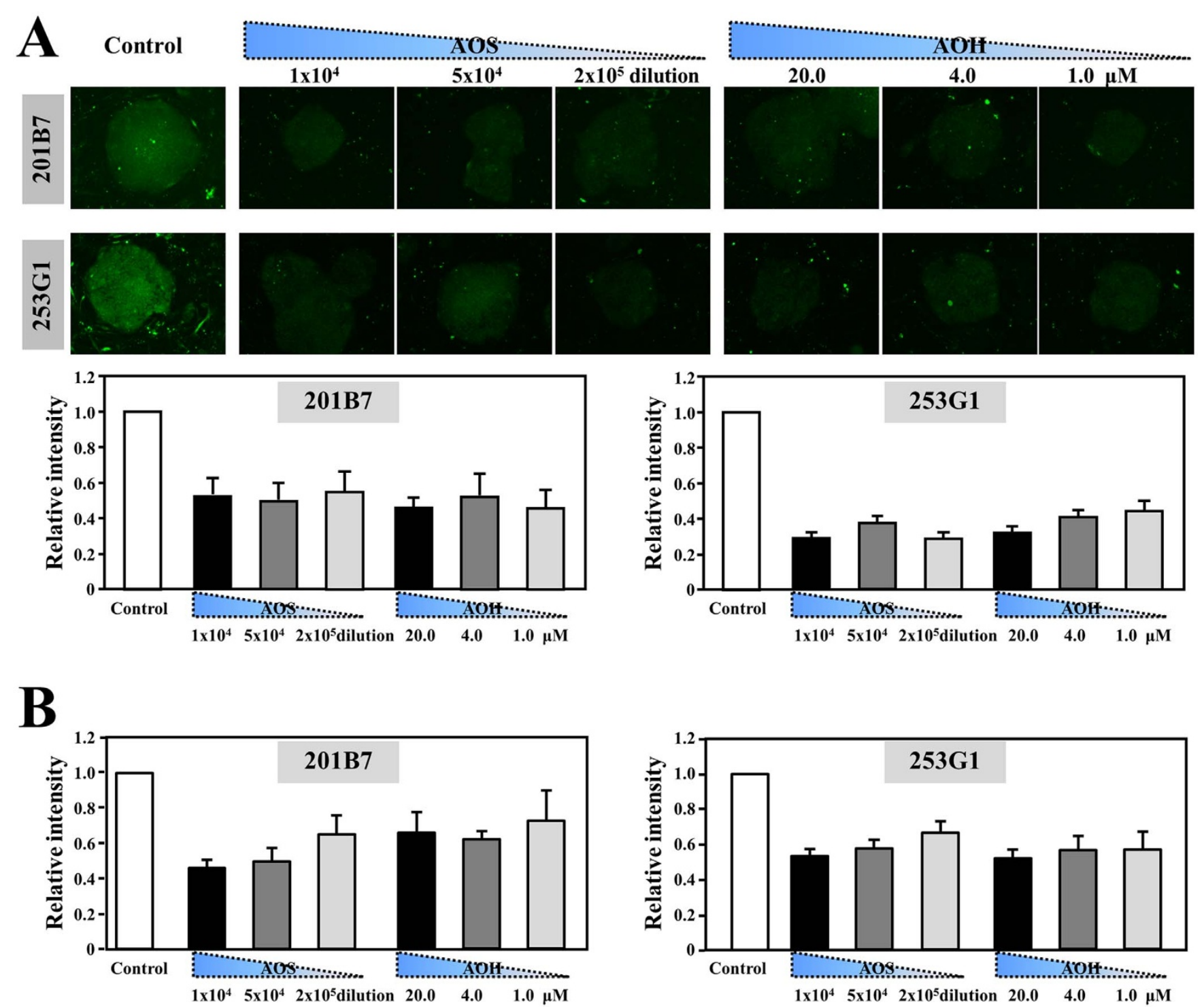

Figure $2 \mid$ Intracellular ROS levels in iPS cells. (A) Intracellular ROS in the iPS cells was loaded with $10 \mu \mathrm{M} 2^{\prime}, 7^{\prime}$-dichlorodihydrofluorescein diacetate for $60 \mathrm{~min}$, and representative images showed relatively lower fluorescence intensity in the iPS cell colonies cultured with antioxidants than that of control. Data of semi-quantitative analysis on the intracellular ROS in 201B7 and 253G1 iPS cells were presented from three separate experiments. (B) The intracellular ROS were also determined by flow cytometry, and data were presented from three separate experiments. Abbreviations: AOS, proprietary antioxidant supplement from Sigma-Aldrich; $\mathrm{AOH}$, Homemade antioxidant cocktail.

stability of iPS cells. We found that the iPS cells grew well and "stemness" was maintained up to 2 months with the addition of low dose antioxidants in medium. Although the addition of low dose antioxidants in culture medium decreased the intracellular ROS levels in iPS cells, it did not affect the expression of 53BP1 and ATM, two critical molecules involved in DNA damage and repair $^{11-13}$. Furthermore, array $\mathrm{CGH}$ analysis indicated that the events of genetic aberrations were decreased only by the supplements with homemade antioxidant cocktail in one of the two tested iPS cell lines.

Free radicals are viewed as harmful by-products of cell metabolism, and it is well known that the accumulation of ROS in cells will induce the oxidation of DNA, lipids, and proteins, which results in cell damage and causes genomic instability. However, a number of studies have identified a critical physiological role of ROS in intracellular signaling ${ }^{14-16}$. We have recently demonstrated that an extreme suppression of ROS by high-dose antioxidants could down-regulate the DNA repair-related protein kinases and conversely causes genomic instability of stem cells ${ }^{9}$. Based on our recent study $^{9}$, a modest inhibition of intracellular ROS by the supplement with low dose antioxidants in medium likely contributes to decrease the DNA damage of human adult tissue stem cells and ES cells cultured in general $\mathrm{CO}_{2}$ incubator $\left(\sim 20 \% \mathrm{O}_{2}\right)$. These findings from past studies pursued us to systemically examine whether low dose antioxidants could improve the quality and genomic stability of iPS cells, one of the most focused stem cell sources for future medical applications.

Data from this study showed that the addition with either 10,000 $\sim$ 200,000-fold diluted proprietary antioxidant supplement or $1 \sim$ $20 \mu \mathrm{M}$ homemade antioxidant cocktail in the culture medium did not affect the growth and "stemness" of iPS cells by 2 months followup, although the additions with antioxidants significantly decreased ROS levels in iPS cells. Strikingly, the decrease of ROS levels in iPS cells by either proprietary antioxidant supplement or homemade antioxidant cocktail did not clearly show a dose dependent manner. This may due to the relative narrow range of antioxidant dosages used for study and the limitation on sensitivity of measuring ROS levels by DCF fluorescence. Otherwise, although the supplements of 
A
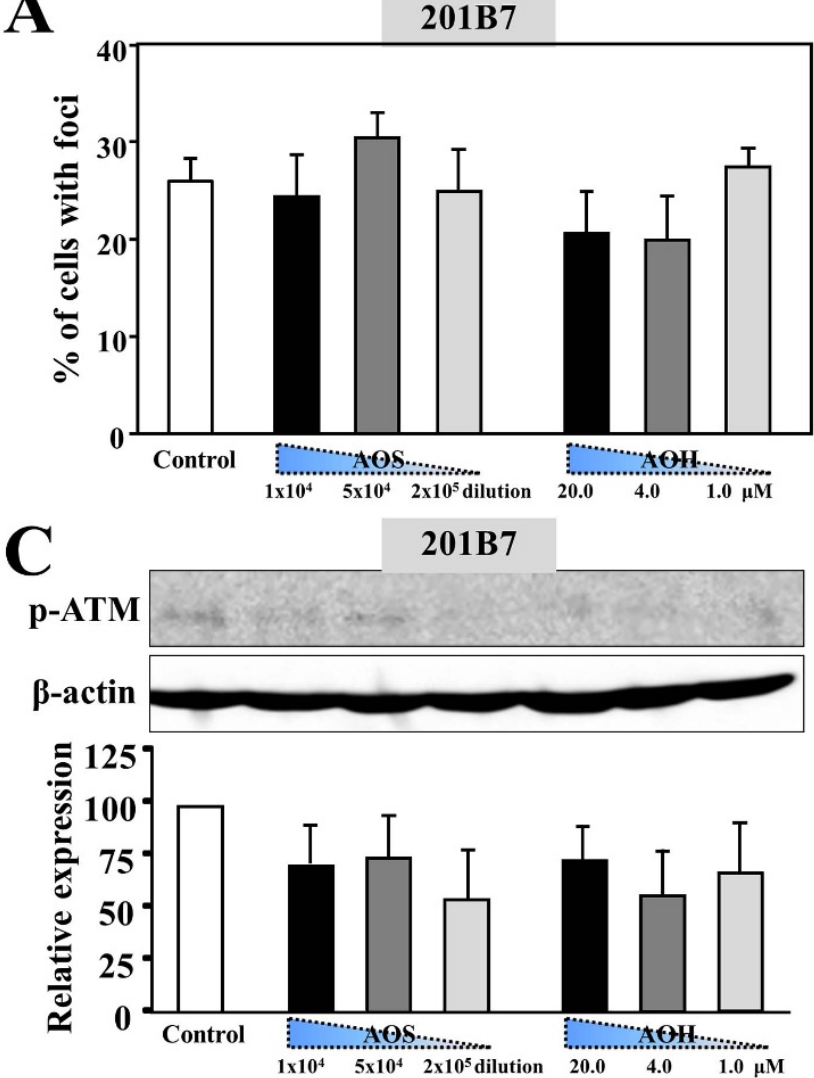

B

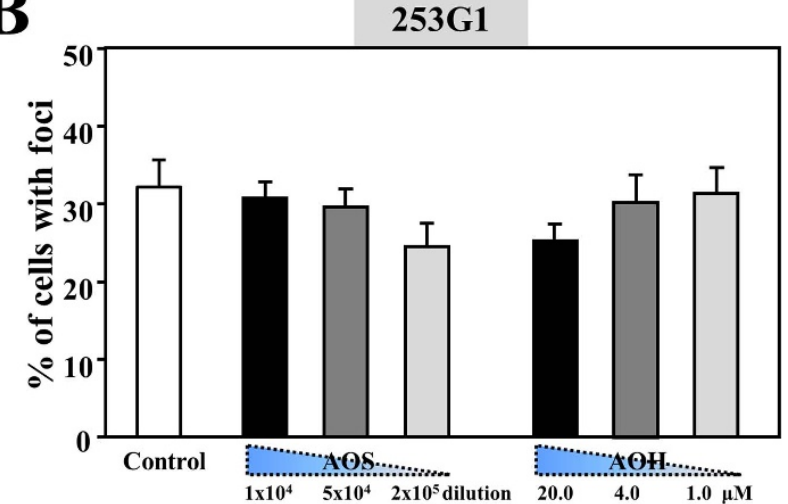

D
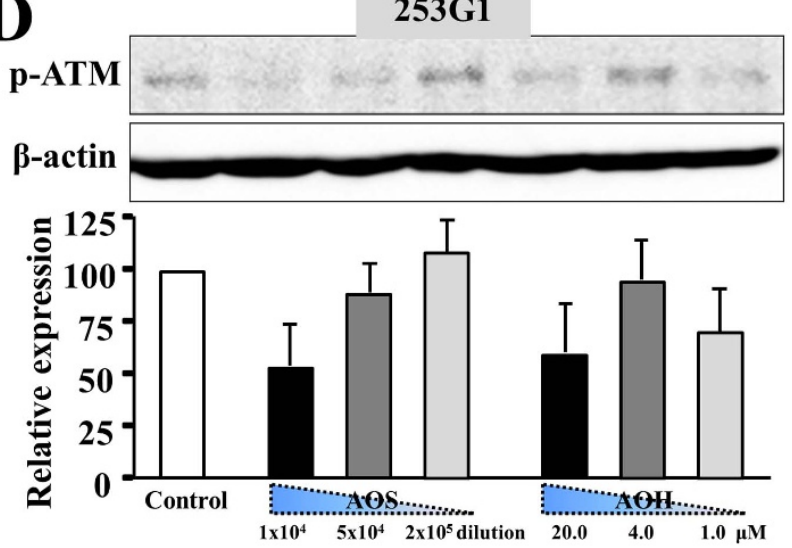

Figure 3 The expressions of 53BP1 and pATM in iPS cells. The expression of 53BP1 was detected by immunostaining, and cells with 53BP1 foci were counted in 201B7 (A) and 253G1 (B) iPS cell lines. The expression of pATM was examined by Western blot. Representative images that cropped from full-length gels (Supplementary Figure 2) was shown, and semi-quantitative analysis of expression was also done (C), (D). The data are presented as the means \pm SD from three separate experiments. Abbreviations: AOS, proprietary antioxidant supplement from Sigma-Aldrich; AOH, Homemade antioxidant cocktail.

A

201B7

อั

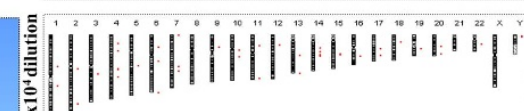

\&

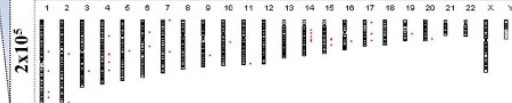

竎

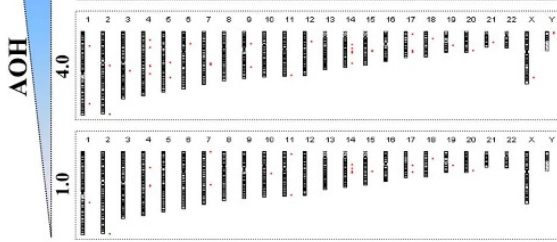

$253 \mathrm{G1}$

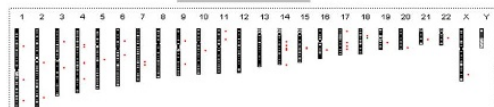

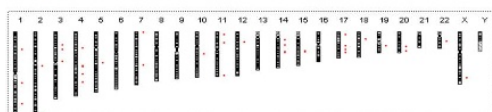

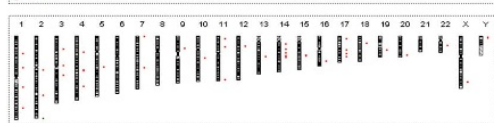

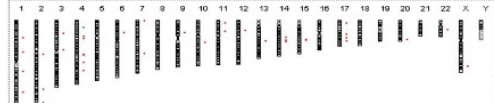

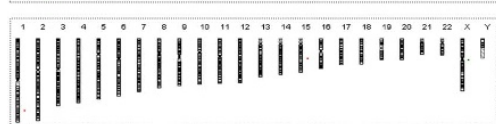

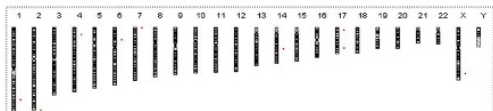

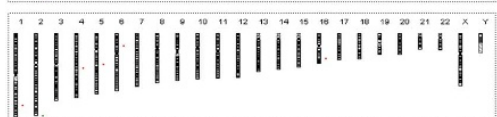

B
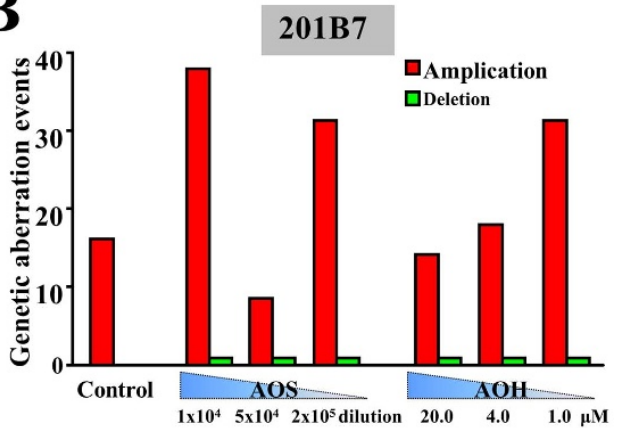

253G1

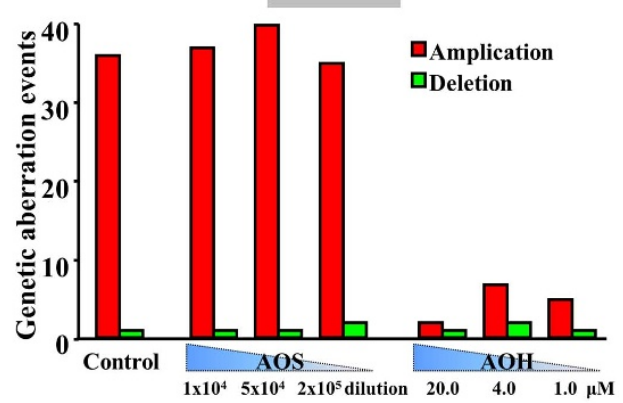

Figure 4 | Array CGH analysis for genetic aberrations in iPS cells after 2 months of culture. (A) With $\log 2$ ratios over 0.75 , the data from array CGH showed some amplification (red dots) and a few of deletion (green dots) in both the 201B7 and 253G1 iPS cell lines cultured with the addition of antioxidants or without. (B) The number of amplification and deletion within the events of genetic aberrations are shown. Abbreviations: AOS, proprietary antioxidant supplement from Sigma-Aldrich; AOH, Homemade antioxidant cocktail. 

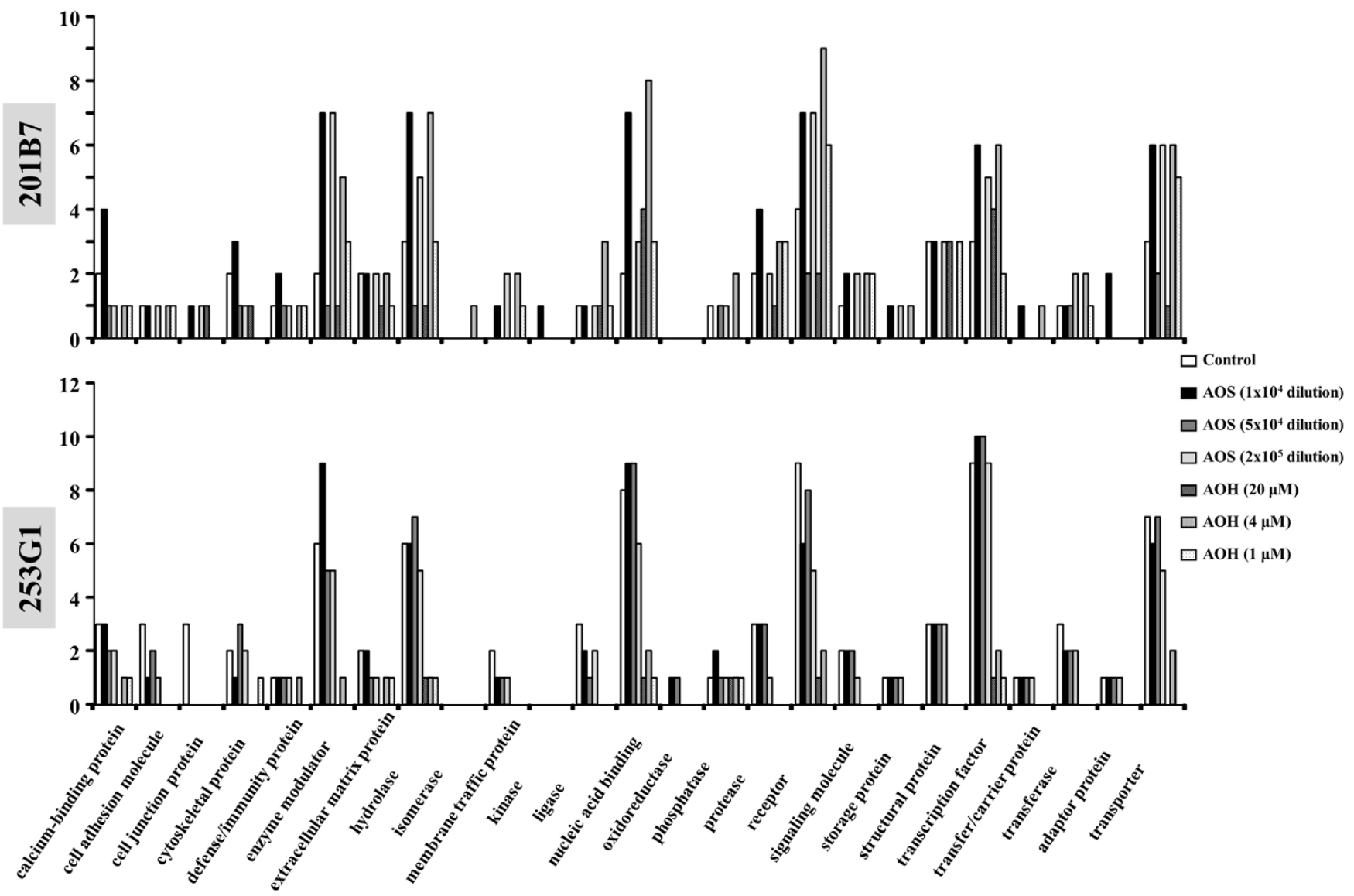

Figure 5 Protein classification of the genetic aberrations detected by array CGH. Most of the increased genetic aberrations were classified as enzyme modulator, hydrolase, nucleic acid binding, receptor, transcription factor, and transporter. Abbreviations: AOS, proprietary antioxidant supplement from Sigma-Aldrich; AOH, Homemade antioxidant cocktail.

low dose antioxidants in medium significantly decreased the intracellular ROS levels in iPS cells to almost $30 \sim 50 \%$ of the control, there was no obviously changes on the expressions of 53BP1 and ATM, indicating that low dose antioxidants had very limited effects on DNA damage and repair for these iPS cells within 2 months of culture.

Chromosomal copy number aberrations are known to be the result of the underlying genetic instability, and array CGH allows the global profiling of such copy number aberrations ${ }^{17}$. Strangely, compared with iPS cells cultured without the addition of antioxidants, array CGH analysis showed that the events of chromosomal copy number aberrations were decreased only in the 253G1 iPS cells supplemented with $1 \sim 20 \mu \mathrm{M}$ homemade antioxidant cocktail. The reason on the variations of genetic aberrations remains unclear, but it may be due to a casually growth selection of iPS cells during passages and a variation between cell lines in response to antioxidants.

Increasing evidences have shown the variation among iPS cell lines, as well as among embryonic stem (ES) cell lines ${ }^{18,19}$. Due to a very strict rule on using human ES cells for study in Japan, we used two different iPS cell lines for experiments to testing the variation. The data of CGH array differed between two iPS cell lines in this study has actually suggested a variation between iPS cell lines. Otherwise, the Primate ES cell Medium (Cat. \#RCHEMD001) used for culturing iPS cells in this study was purchased from company, and the detail recipe of medium was not available due to the highly commercial confidence. Considering the most of medium for stem cell culture consist of antioxidants, the basal level of antioxidants in the Primate ES cell Medium may potential attenuate the oxidative stress-induced damage of iPS cells, which probable partially cancel the protective effects by further addition with either proprietary antioxidant supplement or homemade antioxidant cocktail at a relative low dosages. That might also help to explain why we did not see dose dependence on either ROS levels or genomic stability by the addition of antioxidants in this study.

In all, the addition of low dose antioxidants in culture medium did not obviously affect the growth and "stemness" of iPS cells over 2 months. Although low dose antioxidants moderately decrease the intracellular ROS levels of iPS cells, further experiments with longer term of cultivation will be necessary to confirm the benefit of antioxidants for ex vivo expansion of iPS cells.

\section{Methods}

Long-term culture of human iPS cells. Human iPS cell lines (207B7 and 253G1) purchased from Riken, Japan, were used for this study. The 207B7 iPS cell line was induced by Yamanaka four factors ${ }^{20}$, and the 253G1 iPS cell line was induced by 3 factors without $\mathrm{c}-\mathrm{Myc}^{21}$. These iPS cells were maintained as described previously with a few modifications $\mathrm{s}^{20,21}$. Briefly, iPS cell lines were recovered to 6-well culture plate and incubated in a typical $\mathrm{CO}_{2}$ incubator $\left(95 \% \mathrm{air} / 5 \% \mathrm{CO}_{2}, \sim 20 \% \mathrm{O}_{2}\right)$. After second passage, a single colony of iPS cells was picked and moved into a well of 24 -well culture plate for expansion. The iPS cells expanded from a single colony (passage \#6) were then harvested and initiated to culture with the addition of proprietary antioxidant supplement from Sigma-Aldrich (AOS, Catalogue Number: Sigma A1345) at 10,000-fold, 50,000-fold, and 200,000-fold dilution, and with the addition of homemade antioxidant cocktail $(\mathrm{AOH})$ that consists of $\mathrm{L}$-ascorbate, L-glutathione, and $\alpha$-tocopherol acetate (Sigma-Aldrich) at the concentrations of $20 \mu \mathrm{M}, 4 \mu \mathrm{M}$, and $1 \mu \mathrm{M}$, respectively ${ }^{9}$, or without the addition of any antioxidant as control. We maintained these iPS cells under each condition in parallel for 2 months by regularly passaging (passaged every 5-7 days) and then used for the following experiments (passages \#16 for 207B7 and passages \#14 for 253G1). We used Primate ES cell Medium (Cat. \#RCHEMD001) with the supplement of $5 \mathrm{ng} / \mathrm{mL}$ bFGF (Cat. \#RCHEOT002, ReproCell Inc. Yokohama, Japan) for all culture of the iPS cells, but the feeder cells was prepared by culture mouse embryonic fibroblast in DMEM medium (Sigma-Aldrich) with 10\% fetal bovine serum (Hyclone Laboratories, Inc.). 

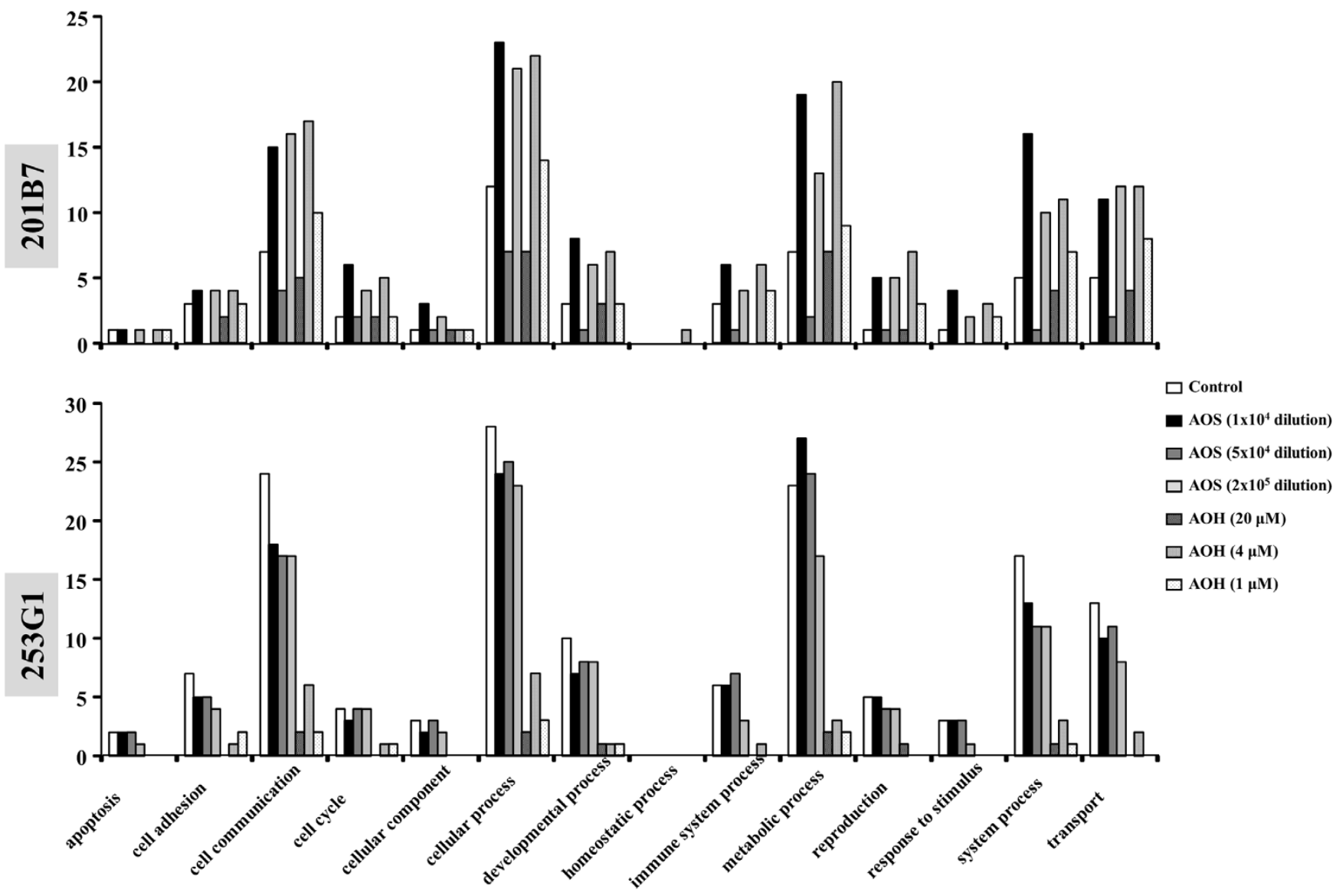

Figure 6 Biological processes affected by the genetic aberrations detected by array CGH. Most of the increased genetic aberrations were associated with cell communication, cellular process, and metabolic process. Abbreviations: AOS, proprietary antioxidant supplement from Sigma-Aldrich; AOH, Homemade antioxidant cocktail.

Determination of the expression of stem cell markers. The "stemness" of iPS cells was estimated by examining the expressions of Oct $3 / 4$, Nanog, and SSEA-4 using immunostaining. Briefly, iPS cells were cultured in 4 -well chamber culture slides (Nalge Nunc International) for 5 days, and then fixed with $1 \%$ formaldehyde for $10 \mathrm{~min}$. After blocking, the cells were incubated with primary antibodies against human Oct3/4, Nanog, and SSEA-4 (R\&D Systems, Inc.) for $1 \mathrm{hr}$ and then with the appropriate Alexa 680 -conjugated secondary antibodies for $20 \mathrm{~min}$. The nuclei were stained with Hoechst 33258. Staining for the expression of ALP was performed using an Alkaline Phosphatase staining kit (Cosmo Bio Co., Ltd).

The expression levels of Oct $3 / 4$ and Nanog were further examined by Western

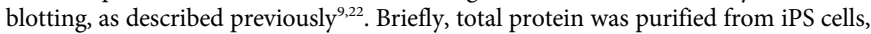
separated using SDS-PAGE gels, and then transferred to nitrocellulose membranes. Membranes were incubated with primary antibodies against Oct3/4, Nanog, or $\beta$ actin, followed by the appropriate horseradish peroxidase-conjugated secondary antibodies, and then visualized using an enhanced chemiluminescence detection kit (Amersham Biosciences).

Determination of intracellular reactive oxygen species (ROS). To detect the intracellular ROS levels, iPS cells were seeded in 4-well culture chamber slides and cultured with or without antioxidants as mentioned above. After approximately 5 days of culture, $10 \mu \mathrm{M} \mathrm{2} 2^{\prime}, 7^{\prime}$-dichlorodihydrofluorescein diacetate (DCFH-DA) (Invitrogen) was added to the cells for $60 \mathrm{~min}^{9,23}$. The cells were then washed, and the intracellular ROS were directly observed as the fluorescence using a fluorescence microscope and were recorded with a digital camera (DP-26, Olympus, Tokyo, Japan). The relative fluorescence intensity was semi-quantitatively measured using Image-Pro Plus software (Media Cybernetics) and normalized by control.

To further quantitative measure the ROS levels, cells cultured in 6-well plates were also added with DCFH-DA for 60 mins, and then trypsin-treated and fixed. The DCF fluorescence intensity in cells was detected by flow cytometer using a FACS Calibur, and data were analyzed with CellQuest software (BD Biosciences) as described previously ${ }^{9,14}$.

Evaluations on DNA damage and repair. To evaluate the DNA damage, iPS cells were seeded on 4 -well chamber culture slides. The cells were fixed in $1 \%$ formaldehyde for $10 \mathrm{~min}$ after 5 days of culture. After blocking, the cells were incubated with primary antibody against 53BP1 (Abcam), followed by a FITC- conjugated secondary antibody. The nuclei were stained with Hoechst 33258. The positively stained cells were observed under fluorescence microscopy with 200 -fold magnification, and more than 200 cells were counted to calculate the percentage of iPS cells with 53BP1 foci in the nucleus ${ }^{24}$.

The expression levels of ATM, a key molecule involved in DNA repair, were measured by Western blotting as described above. Briefly, the total protein was purified from the iPS cells, separated using SDS-PAGE gels, and transferred to nitrocellulose membranes. After blocking, the membranes were incubated with primary antibodies against ATM (phosphorylated at Ser-1981, pATM) or $\beta$-actin, followed by the appropriate horseradish peroxidase-conjugated secondary antibodies. The expression was visualized using an enhanced chemiluminescence detection kit, and semi-quantitative analysis was done by measuring the density of bands using Image J software.

Array comparative genomic hybridization (CGH) and data analysis. An array CGH was performed following the standard Agilent protocol (V7.1). Briefly, genomic DNA (gDNA) was extracted from the iPS cells after 2 months of culture by using the QIAGEN DNeasy Blood \& Tissue kit. Total of $250 \mathrm{ng}$ gDNA samples from iPS cells or $250 \mathrm{ng}$ sex-matched human reference DNA (G1521, Promega) were digested with AluI and RsaI, and then labeled with Cy5- or Cy3-dUTP (SureTag DNA labeling kit, Agilent Technologies), respectively. Following purification with Amicon Ultra columns (Millipore), the labeled DNA yield and dye incorporation were measured using a NanoDrop spectrophotometer (ND-1000, Thermo Scientific). The labeled DNA samples, $2 \mu \mathrm{g}$ human Cot-1 DNA (Agilent Technologies), blocking agent, and Hi-RPM buffer (array CGH Hybridization kit, Agilent Technologies) were mixed together and hybridized at $65^{\circ} \mathrm{C}$ on the standard Agilent $8 \times 60 \mathrm{~K}$ array for 24 hours in a rotisserie oven at $20 \mathrm{rpm}$. The slides were washed and scanned immediately using an Agilent high-resolution scanner. The data were extracted using Agilent Feature Extraction software (version 10.7.1.1) with the CGH_105_Sep09 protocol.

The array CGH data sets were analyzed with the Genomic Workbench 6.5 software (Agilent Technologies). Aberrant regions were determined using the ADM-2 algorithm with the threshold set to 5.0, and the aberration filter was selected with the following parameters: a minimum number of probes in region 3, a maximum of 10,000 aberrations, and a percent penetrance per feature of 0 . A copy number gain was defined as a $\log 2$ ratio $>0.75$, and a copy number loss was defined as a $\log 2$ ratio $<-0.75$. 
Functional categorization of aberrant genes/proteins. To understand the biological significance of the identified chromosome aberrations, the related genes/proteins in the aberrant regions were listed and classified based on the PANTHER (Protein ANalysis THrough Evolutionary Relationships) system (http://www.pantherdb.org), a unique resource that classifies genes and proteins by their functions $\mathrm{s}^{25}$. During this process, the PANTHER ontology, a highly controlled vocabulary (ontology terms) of biological process, molecular function, and molecular pathway, was used to categorize the proteins into families and subfamilies with shared functions.

Statistical analysis. All of the results are presented as the means \pm SD. The statistical significance was determined by 1-way analysis of variance followed by post hoc test (Dr. SPSS II, Chicago, IL). Differences were considered significant when $p<0.05$.

1. Maitra, A. et al. Genomic alterations in cultured human embryonic stem cells Nat. Genet. 37, 1099-1103 (2005).

2. Baker, D. E. et al. Adaptation to culture of human embryonic stem cells and oncogenesis in vivo. Nat. Biotechnol. 25, 207-215 (2007).

3. Sareen, D. et al. Chromosome 7 and 19 trisomy in cultured human neural progenitor cells. PLoS One 4, e7630 (2009).

4. Ivanovic, Z. Hypoxia or in situ normoxia: The stem cell paradigm. J. Cell. Physiol. 219, 271-275 (2009)

5. Ames, B. N., Shigenaga, M. K. \& Hagen, T. M. Oxidants, antioxidants, and the degenerative diseases of aging. Proc. Natl. Acad. Sci. U. S. A. 90, 7915-7922 (1993).

6. van Gent, D. C., Hoeijmakers, J. H. \& Kanaar, R. Chromosomal stability and the DNA double-stranded break connection. Nat. Rev. Genet. 2, 196-206 (2001).

7. Wang, F., Thirumangalathu, S. \& Loeken, M. R. Establishment of new mouse embryonic stem cell lines is improved by physiological glucose and oxygen. Cloning Stem Cells 8, 108-116 (2006).

8. Fehrer, C. et al. Reduced oxygen tension attenuates differentiation capacity of human mesenchymal stem cells and prolongs their lifespan. Aging Cell 6, 745-757 (2007).

9. Li, T. S. \& Marbán, E. Physiological levels of reactive oxygen species are required to maintain genomic stability in stem cells. Stem Cells 28, 1178-1185 (2010).

10. Li, T. S. et al. Expansion of human cardiac stem cells in physiological oxygen improves cell production efficiency and potency for myocardial repair. Cardiovasc. Res. 89, 157-165 (2011).

11. Noon, A. T. \& Goodarzi, A. A. 53BP1-mediated DNA double strand break repair: insert bad pun here. DNA Repair (Amst) 10, 1071-1076 (2011).

12. Kitagawa, R. \& Kastan, M. B. The ATM-dependent DNA damage signaling pathway. Cold Spring Harb. Symp. Quant. Biol. 70, 99-109 (2005)

13. Kinoshita, T. et al. Ataxia-telangiectasia mutated (ATM) deficiency decreases reprogramming efficiency and leads to genomic instability in iPS cells. Biochem. Biophys. Res. Commun. 407, 321-326 (2011).

14. Foreman, J. et al. Reactive oxygen species produced by NADPH oxidase regulate plant cell growth. Nature 422, 442-426 (2003).

15. Niethammer, P., Grabher, C., Look, A. T. \& Mitchison, T. J. A tissue-scale gradient of hydrogen peroxide mediates rapid wound detection in zebrafish. Nature 459, 996-999 (2009).

16. Owusu-Ansah, E. \& Banerjee, U. Reactive oxygen species prime Drosophila haematopoietic progenitors for differentiation. Nature 461, 537-541 (2009).
17. Costa, J. L., Meijer, G., Ylstra, B. \& Caldas, C. Array comparative genomic hybridization copy number profiling: a new tool for translational research in solid malignancies. Semin. Radiat. Oncol. 18, 98-104 (2008).

18. Robinton, D. A. \& Daley, G. Q. The promise of induced pluripotent stem cells in research and therapy. Nature 481, 295-305 (2012).

19. White, M. P., Rufaihah, A. J., Liu, L., Ghebremariam, Y. T., Ivey, K. N., Cooke, J. P. \& Srivastava, D. Limited gene expression variation in human embryonic stem cell and induced pluripotent stem cell-derived endothelial cells. Stem Cells 31, 92-103 (2013).

20. Takahashi, K. et al. Induction of pluripotent stem cells from adult human fibroblasts by defined factors. Cell 131, 861-872 (2007).

21. Nakagawa, M. et al. Generation of induced pluripotent stem cells without Myc from mouse and human fibroblasts. Nat. Biotechno. 26, 101-106 (2008).

22. Kawakatsu, M., Goto, S., Yoshida, T., Urata, Y. \& Li, T. S. Nuclear translocation of glutathione S-transferase $\pi$ is mediated by a non-classical localization signal. Biochem. Biophys. Res. Commun. 411, 745-750 (2011).

23. Yoshida, T., Goto, S., Kawakatsu, M., Urata, Y. \& Li, T. S. Mitochondrial dysfunction, a probable cause of persistent oxidative stress after exposure to ionizing radiation. Free Radic. Res. 46, 147-153 (2012).

24. Kawakatsu, M. et al. Nicaraven attenuates radiation-induced injury in hematopoietic stem/progenitor cells in mice. PLoS One 8, e60023 (2013).

25. Mi, H., Guo, N., Kejariwal, A. \& Thomas, P. D. PANTHER version 6: protein sequence and function evolution data with expanded representation of biological pathways. Nucleic Acids Res. 35, D247-252 (2007).

\section{Acknowledgments}

This study was supported by a Grant-in-Aid from the Ministry of Education, Science, Sports, Culture and Technology, Japan, and by Uehara Memorial Foundation. The founders did not participate in this study.

\section{Author contributions}

H.X., K.H. and T.L. conceived and designed the experiments. L.L., M.K., C.G., Y.U., W.H., H.A., H.D., Y.K., T.T., S.G., Y.O., T.L. performed the experiments and analyzed the data. T.L. and L.L. wrote the main manuscript text. All authors reviewed the manuscript.

\section{Additional information}

Supplementary information accompanies this paper at http://www.nature.com/ scientificreports

Competing financial interests: The authors declare no competing financial interests.

How to cite this article: Luo, L. et al. Effects of antioxidants on the quality and genomic stability of induced pluripotent stem cells. Sci. Rep. 4, 3779; DOI:10.1038/srep03779 (2014)

cc)(-) $\odot$ This work is licensed under a Creative Commons Attribution-

By No No NonCommercial-NoDerivs 3.0 Unported license. To view a copy of this license visit http://creativecommons.org/licenses/by-nc-nd/3.0 